Royal Netherlands Institute for Sea Research

This is a postprint of:

Liu, Q.X., Rietkerk, M., Herman, P.M.J., Piersma, T., Fryxell, J.M. \& van de Koppel, J. (2016). Phase separation driven by density-dependent movement: A novel mechanism for ecological patterns. Physics of Life Reviews, 19, 107-121.

Published version: $\underline{\text { dx.doi.org/10.1016/j.plrev.2016.07.009 }}$

Link NIOZ Repository: www.vliz.be/nl/imis?module=ref\&refid=282880

[Article begins on next page]

The NIOZ Repository gives free access to the digital collection of the work of the Royal Netherlands Institute for Sea Research. This archive is managed according to the principles of the Open Access Movement, and the Open Archive Initiative. Each publication should be cited to its original source - please use the reference as presented.

When using parts of, or whole publications in your own work, permission from the author(s) or copyright holder(s) is always needed. 
Running title: Movement-driven pattern formation

\section{Corresponding author:}

Prof. Dr. Quan-Xing Liu, State Key Laboratory of Estuarine and Coastal Research, East China Normal University, 200062 Shanghai, P.R. China; Tel: 86-21- 62224942; Email: qxliu@sklec.ecnu.edu.cn

Prof. Dr. Johan van de Koppel, Department of Estuarine and Delta Systems, Royal Netherlands Institute for Sea Research and Utrecht University, PO Box 140, 4400 AC Yerseke, The Netherlands; Tel: 31113 577455; Email: Johan.van.de.Koppel@nioz.nl.

\section{Research area:}

\section{Interdisciplinarity, Ecology, Spatial Self-Organization}

Keywords: self-organization; phase separation; density-dependent movement; collective behaviour

\section{Highlights:}

- Phase separation is introduced as a universal mechanism for movement-driven self-organized patterning in ecology.

- Density-dependent movement appears to be a ubiquitous process in a wide variety of aggregating organisms, explaining their aggregation and pattern formation.

- We provide guidelines to integrate the physical principle of phase separation within ecological studies on the movement and aggregative behavior of animals.

\section{More detailed information:}

Number of words in abstract and main text: 200 and 8101 words respectively.

Number of cited references: 126 references.

Number of tables \& figures: 1 table and 5 figures.

The type of article:

Reviews and Syntheses 


\title{
Phase separation driven by density-dependent movement: a novel mechanism for ecological patterns
}

Quan-Xing Liu ${ }^{1,2,8,}{ }^{*}$, Max Rietkerk ${ }^{3}$, Peter M.J. Herman ${ }^{2}$, Theunis Piersma ${ }^{4,5}$, John M. Fryxell $^{6}$, and Johan van de Koppel ${ }^{2,7, *}$

\author{
*Corresponding author: \\ Prof. Dr. Quan-Xing Liu \\ State Key Laboratory of Estuarine and Coastal Research, East China Normal University, \\ 200062 Shanghai, P.R. China; Tel: 86-21-62224942; Email: qxliu@sklec.ecnu.edu.cn \\ Prof. Dr. Johan van de Koppel, \\ Department of Estuarine and Delta Systems, Royal Netherlands Institute for Sea \\ Research and Utrecht University, PO Box 140, 4400 AC Yerseke, The Netherlands, \\ Email: Johan.van.de.Koppel@nioz.nl.
}

\section{Affiliations:}

1. State Key Laboratory of Estuarine and Coastal Research, East China Normal University, 200062 Shanghai, P.R. China;

2. Department of Estuarine and Delta Systems, Royal Netherlands Institute for Sea Research and Utrecht University, PO Box 140, 4400 AC Yerseke, The Netherlands;

3. Department of Environmental Sciences, Copernicus Institute, Utrecht University, P.O. Box 80155 TC Utrecht, The Netherlands;

4. Department of Coastal Systems, NIOZ Royal Netherlands Institute for Sea Research, 1790 AB Den Burg, The Netherlands;

5. Animal Ecology Group, Centre for Ecological and Evolutionary Studies, University of Groningen, PO Box 11103, 9700 CC Groningen, The Netherlands;

6. Department of Integrative Biology, University of Guelph, 50 Stone Road East, Guelph, Ontario N1G 2W1, Canada;

7. Groningen Institute for Evolutionary Life Sciences, University of Groningen, PO Box 11103, 9700 CC Groningen, The Netherlands;

8. Center for Global Change and Ecological Forecasting, East China Normal University, Shanghai 200062, P.R. China. 


\begin{abstract}
1 Many ecosystems develop strikingly regular spatial patterns because of small-scale

2 interactions between organisms, a process generally referred to as spatial self-

3 organization. Self-organized spatial patterns are important determinants of the

4 functioning of ecosystems, promoting the growth and survival of the involved organisms,

5 and affecting the capacity of the organisms to cope with changing environmental

6 conditions. The predominant explanation for self-organized pattern formation is spatial

7 heterogeneity in establishment, growth and mortality, resulting from the self-organization

8 processes. A number of recent studies, however, have revealed that movement of

9 organisms can be an important driving process creating extensive spatial patterning in

10 many ecosystems. Here, we review studies that detail movement-based pattern formation

11 in contrasting ecological settings. Our review highlights that a common principle, where

12 movement of organisms is density-dependent, explains observed spatial regular patterns

13 in all of these studies. This principle, well known to physics as the Cahn-Hilliard

14 principle of phase separation, has so-far remained unrecognized as a general mechanism

15 for self-organized complexity in ecology. Using the examples presented in this paper, we

16 explain how this movement principle can be discerned in ecological settings, and clarify

17 how to test this mechanism experimentally. Our study highlights that animal movement,

18 both in isolation and in unison with other processes, is an important mechanism for

19 regular pattern formation in ecosystems.
\end{abstract}


22 When left undisturbed, ecosystems can develop striking regular spatial patterns and

23 patchiness, caused by the interactions among organisms or between organisms and their

24 environment $[1,2]$. Such self-organized spatial patterns have been found in a wide range

25 of ecosystems, ranging from arid bush lands to marine corals [2-4]. So far, most studies

26 explaining spatial patterns in ecosystems highlight spatial variation in processes such as

27 growth, birth, mortality or respiration, as the driving process (from hereon called growth-

28 based processes). This variation is considered to result from the interplay of multiple

29 interactions between organisms operating at different spatial scales. For example,

30 localized facilitation between individuals in combination with large-scale competition for

31 resources generates regular spatial patterns in for instance bushland vegetation just south

32 of the Sahara as well as in mussel beds in the Wadden Sea [5-9]. Self-organized spatial

33 patterns are considered key to understanding ecological stability [10], diversity [1] and

34 ecosystems functioning [7, 9, 11-14]. Hence, understanding of the processes driving

35 pattern formation is important to understand how these systems may respond to, for

36 instance, climate change.

37 A multitude of studies have linked regular pattern formation in ecosystems to the 38 activation-inhibition principle developed by Alan Turing [15] and Hans Meinhardt [16,

39 17]. This general mathematical principle proposes that the interaction between a self40 amplifying activation process operating at small spatial scales and an inhibiting process

41 that operates at a larger spatial scale can explain pattern formation in a wide variety of

42 chemical and morphological systems (see Box 1). Essential here are a diffusive or 43 differential flow instability, meaning that the rate of spread of the inhibitor - either 
44 through diffusion or through flow - significantly exceed that of the activator. This causes

45 the effect of the activator to remains local, while the inhibitor exerts its effect also at

46 larger distance. As a result of this, there is a scale-dependent feedback of organisms on

47 the growth of neighboring conspecifics. Locally, there is a positive feedback, where

48 organisms promote each other's growth, but at a larger distance a negative effect on

49 growth predominates.

50 Recent papers highlight the importance of adaptive animal behavior in explaining 51 patterns observed in nature [12, 18-25]. Here, animals aggregate to form concentrated 52 groups, clumps, herds, or schools in order to avoid predation, dislodgement of other 53 losses they face increasingly when alone. Within the groups, they experience lower 54 chances of predation by means of dilution of predator attention, can confuse predators in 55 isolating an individual, or can benefit from the vigilance of others in the group and 56 thereby improve food intake [26]. To form groups, animals must adjust their movement 57 and other forms of behavior, in the first place by moving towards others when they are 58 alone, but most importantly by adjusting their movement speed when they have merged 59 to form a group [12, 24, 27-29]. This adaptive behavior is essentially density-dependent, 60 as it adjusts to the local density of conspecifics. Density-dependent movement can lead to 61 the formation of groups that, in combination with constrains on the total number of 62 animals, leads to regular or semi-regular distribution of groups in the landscape [29]. This 63 mechanism of pattern formation is fundamentally different from Turing's activator 64 (facilitation) - inhibition mechanism. Rather than organisms affecting each other's growth 65 rates, this mechanism centers around animal behavior, where animal movement adjusts to 66 variation in density, amplifying this variation to form aggregations that in turn form 
67 coherent spatial patterns (see Table 1 for a summary for different ecosystems). Although

68 the implications of density-dependent movement for population dispersal has been treated

69 in studies going back to the classic work on clines and spread [30-34], it has received

70 little attention as a general mechanism of self-organization in ecological theory.

71 In this paper, we review studies on movement-driven self-organization in ecological

72 systems. We show that many of recent studies adhere to the physical principle of 'phase

73 separation' [35], either in a direct mathematical way [24, 29, 36, 37] or by revealing the

74 characteristic movement patterns. So-far unrecognized in ecology, this principle explains

75 movement-driven self-organization in a wide range of physical [28, 29, 38, 39] and

76 chemical systems [40, 41] and as we will argue, also ecological systems [24]. To keep the

77 scope of the paper manageable, we only consider the mechanisms that could reasonably

78 be termed as 'density-dependent' movement, and that deal with self-organization

79 dynamics. First we briefly explain the limitations of the traditional models of spatial

80 population dynamics that can be found in many textbooks $[4,31,42]$. We then review a

81 number of studies on a diverse group of species that highlight how density-dependent

82 dispersal can generate aggregation and - in some occasions - regular pattern formation.

83 We discuss the conditions under which phase separation occurs and whether these

84 conditions are likely to be common. We limit ourselves to the processes of animal

85 aggregation and group formation, and will not extensively discuss the behavior and

86 coordination of animal movement within groups, which is extensively discussed in the

87 literature on collective animal movement [43]. 
90 Models describing the spatial dynamics of animal populations often describe dispersal of

91 animals using a diffusion term that follows Fick's law [44]:

$92 \frac{\partial N}{\partial t}=f(N, R)+D \nabla^{2} N$.

93 Here, $N$ is the local density of the population, $f(N, R)$ summarizes all local growth, birth

94 and mortality processes as a function of local densities of $N$ and other factors $R$ such as

95 food availability, $D$ is a diffusion coefficient, and $\nabla^{2}$ is the Laplace operator. This

96 formulation implicitly presumes that individual movement can be approximated by

97 random, Brownian motion [45]. A fundamental characteristic of the use of diffusion is

98 that in the absence of population growth or mortality, animal movement always leads to

99 dispersion, i.e. leads to an even distribution of the population over its spatial range. For

100 concentrations of animals to form, the term $f(N, R)$ only needs to be slightly spatially

101 heterogeneous at the starting, i.e. growth conditions need to vary over the landscape, as is

102 the case in Turing's model. Hence, this general formulation of population dynamics is

103 unable to describe aggregative movement by the animals, and hence precludes group

104 formation of in animals in the form of clumps, schools or herds.

\section{A physical principle for movement-driven pattern formation}

106 In the physical and chemical sciences, it is widely recognized that aggregation of atoms

107 and molecules can lead to the formation of self-organized patterns, a process called 'phase

108 separation' $[35,41]$. Phase separation describes the spontaneous separation of mixed

109 fluids [38], such as molten alloys, into their separate components [39]. During this 
110 process, spatial patterns are at first often fine-grained, but they grow coarser as time

111 progresses due to spatial separation of the components of the alloys. The physical

112 principle of phase separation has been captured by a simple partial differential equation

113 by Cahn and Hilliard in 1958 [35]. Under this scenario, the dynamics of the density field

114 is governed by

$115 \frac{\partial N}{\partial t}=D \nabla^{2}\left(f(N)-\kappa \nabla^{2} N\right)$,

116 where $f(N)$ describes the energetic state of $N$. reflecting its tendency to move in response

117 to local variation in density, and $\kappa$ accounts the nonlocal diffusivity or tension in ecology

118 and physics respectively. Essential to the Cahn-Hilliard $(\mathrm{CH})$ principle is a switch from

119 dispersive to aggregative movement of particles as the local concentration of similar

120 particles increases. In other words, particles move towards concentrations of similar

121 particles, amplifying the formation of these aggregations. The Cahn-Hilliard model has

122 become the standard description of phase separation driving pattern formation in metal 123 alloys [39, 41, 46], fluid flow [40, 47], and microbiology [28, 48]. Moreover, recent

124 developments in theoretical physics expand this principle to "self-propelled particles", e.g

125 the movement of (often microbial) organisms [28, 29, 36, 49-51]. Here, a number of

126 studies argue that active Brownian particles, e.g., a bacterial or algal suspension, can

127 spontaneously separate into a concentrated and a dilute phase resulting from the 128 movement behavior of the microbes [27, 28, 38].

129 Central to the explanation put forward by the Cahn-Hilliard model is a density-dependent

130 relation between particle movement and the local concentration or density of similar

131 particles. In this review, we highlight that this phenomenon is also very general to 
132 ecological systems, where animals adjust their movement speed directly or indirectly to

133 the behavior of conspecifics. Here, it can lead to a wide range of aggregative phenomena,

134 ranging from the formation of regular patterns, to animal concentrations such as herds

135 and fronts, and to highly organized phenomena such as the collective movement of

136 organisms as swarms of birds or schools of fish. Below, we review examples of each of

137 these three categories.

138 Regular pattern formation driven by density dependent movement

139 Mussel beds. The blue mussel (Mytilus edulis) is a common filter-feeding animal living

140 on rocky shores and intertidal flats. Mussels develop net-shaped or clumped spatial

141 patterns at small spatial scales (less than $1 \mathrm{~m}$ ), especially in mussel beds on soft

142 sediments (Fig.1a). Laboratory experiments demonstrate that both net-shaped and

143 clumped patterns can develop from a homogenous initial distribution due to a behavioral

144 response of the mussels to the local density of conspecifics [12]. Mussels move fast at

145 both low and high density, but move much slower at intermediate densities. This leads to

146 aggregation into clumps of intermediate size. Laboratory experiments, lasting for only a

147 day, highlight that pattern formation at the clump scale is a purely movement-driven

148 process; no change in mussel numbers occurs within these short experimental timescales.

149 This process of behavioral self-organization, explaining the emergence of regularly

150 distributed mussel clumps, is adaptive in that it simultaneously reduces predation and

151 dislodgment risks, and minimizes competition for algae [12].

152 Interestingly, our recent study of the aggregative movement of mussels demonstrates that 153 the Cahn-Hilliard principle can be applied to spatial pattern formation in mussels [24]. 
154 Results from quantitative experiments of mussel movement revealed that mussel

155 movement speed decreases in a characteristic fashion with local mussel density (Fig.1b).

156 Mathematical analysis revealed that a decrease of movement speed at intermediate

157 density translates into a shift from dispersive movement at low mussel density to

158 aggregative movement at intermediate density, which is an essential condition for pattern

159 formation in the Cahn-Hilliard principle. We showed that the model of density-dependent

160 aggregation and pattern formation in mussels is mathematically similar to the original

161 model of Cahn and Hilliard [24]. Hence, aggregation and pattern formation in mussels is

162 a process that is equivalent to the familiar process of phase separation in physics.

163 Ants. Movement-driven self-organization processes are an important factor in the

164 building of spatial structures by social insects [18, 52]. For instance, the ant Messor

165 sancta creates regular patterns of ant corpses within ant cemeteries, a specific place

166 where they gather their dead corpses. This process is completely spontaneous, as there is

167 no prior information, nor a leader, among ants. A single cluster (pile) is gradually built as

168 the result of a collective effort, where ants pick up a corpse from a small pile of dead

169 bodies and then deposit the corpse in an already existing cluster. Experimental

170 observation of ant behavior shows that ants pick up or drop corpses with a probability

171 that increases with the local density of corpses, as shown in Figure 1d. The unladen ants

172 pick up corpses with a probability that significantly declines at larger cluster sizes,

173 whereas corpse-carrying ants drop corpses with a probability that increases with cluster

174 size [18]. In addition, ants drop corpses with a fixed probability outside piles, leading to a

175 limited range of transport. If the size of the experimental arena is limited, or if the area

176 contains spatial heterogeneities, ordered clusters will develop along the borders of the 
177 arena or more generally along the heterogeneities [18, 53]. Such a self-organized pattern

178 is shown in Fig.1c. Similar to the mussel example, movement is the dominant process of

179 pattern formation, as corpses are aggregated by density-dependent movement of the ants.

180 Here, the chance of movement is a function of the local density of other corpses, making

181 the net movement density-dependent.

182 Slime molds. Movement plays likewise an important role in the formation of fruiting

183 bodies in colonies of the cellular slime mold, Dictyostelium discoideum. D. discoideum is

184 a social soil amoeba with a complex life cycle. It morphs from a collection of unicellular

185 amoebae into a multicellular slug, and finally into a fruiting body [54]. The movement of

186 the cells of $D$. discoideum during the aggregation and the migration stages is controlled

187 by cAMP, which is produced by the cells themselves [55-57]. At an early stage a few

188 scattered cells spontaneously secrete a single pulse of cAMP (cyclic adenosine

189 monophosphate), followed by cells in the surrounding vicinity secreting more cAMP.

190 This disturbance initially triggers the movement of $D$. discoideum into a cellular

191 aggregation, later to form connected banding that migrates towards the higher

192 concentration of cAMP, like a travelling wave [58]. Cells in dense concentrations

193 produce more cAMP, leading to a higher rate of aggregation for amoeba until saturating

194 (setting a maximal speed) at high cell density [55, 59]. Hence, the underlying mechanism

195 of aggregative movement is indirectly controlled by local cell densities. The typical

196 patterns are spiral waves in the cAMP concentrations and branching patterns in $D$.

197 discoidenum cells as shown in Fig.1e, where the spiral wave propagates outwards, the 198 cells move inwards $[55,58]$. 
199 Bacteria. The bacterium Escherichia coli and similarly other bacteria, may form a wide 200 variety of colony structures ranging from arrays of spots to radially oriented stripes and

201 arrangements of more complex elongated spots [60,61]. Alternative patterns include

202 regular stripes and circular rings when bacteria are grown in a matrix of agar with the

203 inducer anhydrotetracycline [62]. The traditional explanation of pattern formation in

204 bacteria is that it is based on chemotactic behaviour [63, 64]. Bacteria emit a

205 chemoattractant when they experience oxidative stress conditions. Bacterial cells are

206 thought to sense this chemical and swim up a gradient, creating spatial concentrations of

207 bacterial cells, after which eventually the bacteria turn nonmotile, freezing the pattern

208 into space. However, recent studies show that the key process explaining aggregation is

209 deceleration of movement at high cell density $[27,48,62,65,66]$. It can be captured by

210 density-dependent mobility, where the swimming speed of E. coli changes with the

211 density of conspecifics in the local environment. This process will lead to a net cell flow

212 toward the high density region and spatial pattern formation (Fig.1f).

\section{Herds and schools: mobile aggregations}

214 The formation of aggregations such as schools of fish or herds of mammals is very

215 general in nature as an anti-predator defense, and is, like the mussel system, driven by

216 movement. Hence, beyond providing a general principle for the formation of regular

217 patterns as was described in the above mentioned examples, the principle of phase

218 separation may extend to aggregation in mobile groups such as flogs of birds, herds of

219 grazing mammals, and schools of fish, which are known to aggregate in response to

220 mobile predators [67-70]. Below, we review studies that reveal that - similar to mussels

221 and ants - density-dependent movement is a crucial mechanism behind the formation of 
such mobile groups.

223 Elk. The elk (Cervus elaphus canadensis) is one of the largest and most widespread land

224 mammals in North America, Europe, and eastern Asia. Both sedentary and migratory

225 populations have been repeatedly identified, often within the same ecosystem [71, 72]. In

226 contrast to the classic correlated random movement used in many population models, elk

227 movements have been clearly demonstrated to be density-dependent [73, 74]. Elk with

228 GPS satellite radio-collars that were introduced into a novel ecosystem were studied over

229 time in relation to group structure and group status (Fig. 2a). Haydon et al. [75] and Yott

230 et al. [74] demonstrate that elk movement is driven by two important processes, described

231 as fission (individuals leaving groups) and fusion (individuals joining up with a group)

232 respectively. Ungrouped elk individuals were found to move greater distance than

233 grouped animals, grouped animals were more likely to leave small groups than larger

234 groups, and single individuals were more likely to join nearby groups than those that

235 were more distant [75], shown as in Figure 2c and 2d. As dispersing individuals spread

236 out from the initial introduction site, they randomly encountered clusters of other elk

237 individuals. Over a matter of weeks the rate of population spread slowed appreciably [73],

238 but more importantly small clusters of individuals coalesced into local herds. Hence,

239 similar to the other examples, density-dependent rates of movement by elk rapidly led to

240 group formation and spatial patterning across the landscape.

241 Logically, the movement of aggregated (e.g., herd-forming) consumers is not only

242 dependent on the density of conspecifics, but also on the density of their food. Here, they

243 move more extensively when food density is low. In this case, the formation of mobile

244 animal aggregation such as herds and schools due to density-dependent movement may 
245 have important complications for the stability of grazing systems. While aggregation of

246 mussels has no immediate consequence for food availability because of continued supply

247 of algae by the tidal water, herds of grazing mammals can locally deplete their sessile

248 food. Hence, there is an immediate necessity to move as a group to avoid food depletion.

249 This behavior has been observed in herds of grazers, for instance in the Serengeti

250 ecosystems [76-79], where ungulates aggregate as an anti-predation adaptation, and move

251 as a front to through the Serengeti plains as shown in Fig.2b. The movement of individual

252 wildebeest dramatically slows once they encounter ungrazed lands. Dense wildebeest

253 herds rapidly deplete the grass resources available locally, leading to continual relocation

254 of groups across the Serengeti Plains [80]. This complex combination of resource and

255 conspecific-dependent movement behavior results in transient aggregations of wildebeest

256 in extensive grazer fronts that track seasonal changes in the spatial gradient of food

257 availability, often termed the green wave [77, 78, 81-84]. This aggregative process helps

258 stabilize an otherwise unstable grazing interaction [85, 86]. Studies of other species show

259 that restriction of group movement by vegetation [82, 83] or immediate danger of

260 predation when moving, can cause local depletion of food sources leading to the

261 formation of grazing fronts that can strongly destabilize consumer-resource interactions

262 [87], such as those observed in snail-grazed salt-marshes [88] and urchin-grazed kelp

263 beds [89].

\section{Collective animal movement}

265 Density-dependent movement can also generate remarkably complex patterns when

266 animals not only influence each other's rate of movement, but also directionality. This has

267 been studied extensively in the field of collective animal behavior, a field that studies the 
268 joint movement of animals in schools and flocks. For instance, using density-dependent

269 movement features, Farrell et al [90] and Buhl et al [91] showed that a group of

270 individuals (locusts) that align their direction and patterns with that of their neighbors

271 undergo a rapid transition from random motion to coherent motion where all individuals

272 move in a common direction to form spotted and striped patterns. In parallel,

273 biophysicists [92-95] showed that collective cell migration in 2-dimensional cultures

274 reveals a similar density-dependent movement, when beyond a threshold density. These

275 theoretical and experimental studies demonstrate that density-dependent movement is a

276 universal ecological feature generating a multitude of complex spatial configurations, not

277 only regular patterns. Moreover, this work suggests that collective changes in the

278 orientation of individuals within groups could potentially be just as profound as density-

279 dependent changes in movement velocity in generating spatial patterning. It seems quite

280 likely both behavioral processes are relevant in explaining pattern formation in mobile

281 group-forming organisms.

282 Although many studies imply that density-dependent relationships exist in collective 283 behavior [90, 92, 94-96], direct experimental verification remains elusive. A very 284 interesting example has recently emerged in a study on Sperm movement. Sperm cells 285 race via their flagella to reach an egg in a viscous medium and the first one to get there 286 will be able to fertilize it [97]. A solitary sperm cell is more vulnerable to deviations from 287 a straight trajectory owing to the rotational fluctuations caused by imbalanced torque 288 exerted by their flagellum, whereas sperm can group together to form cooperative 289 bundles that make it easier for them to swim along straighter paths. Sperm groups have 290 been observed in several vertebrate and invertebrate taxa, ranging from sperm pairs to 
291 massive aggregates containing hundreds of sperm [98, 99]. Using the fine-scale imaging

292 of spermatozoa of deer mice, Fisher et al $[100,101]$ have revealed that the sperm cells in

293 the rodent genus Peromyscus form motile aggregations of 2-30 cells by adhering to one

294 another at sperm head or head hook to midpiece (See Fig.3a). The increased number of

295 sperm cells within an aggregated cluster significantly increased group average velocity

296 because the group exhibits greater linearity in its movement path, without an increase in

297 speed. Hence, sperm cooperation allows them to migrate more quickly through the

298 female reproductive tract. The average movement velocity would be expected to decrease

299 once the clusters grew larger than seven cells, due to sperm cells swimming against each

300 other, a prediction consistent with density-dependent velocity measurements (Fig. 3b).

301 Obviously, this process does not adhere to the phase separation principle, as movement is

302 maximal at intermediate densities, but is highlights that density-dependent movement is a

303 crucial element in a variety of complex aggregative phenomena.

\section{Further examples of density-dependent movement}

305 Empirical evidence suggests that density-dependent movement is a common phenomenon

306 in ecology. An extensive literature exists on density-dependent movement in animals [31,

307 33, 34, 102]. However, little attention has been given into how density-dependent

308 movement affects spatial self-organization processes. Density-dependent movement rates

309 have been recorded in social insects such as aphids and beetles [103, 104] and even

310 zooplankton $[105,106]$. Empirical data of aphid movement were obtained using marked

311 aphids varians on fireweed flower stalks. The data show that settlement of moving aphids

312 increased as a function of local aphid density during the first 6-8 hours following aphid

313 released. The probability of termination of movement by the aphids increased by more 
314 than an order of magnitude as the local density increased from 1 to 40 individuals (see

315 Fig. 4a). Similar to the aphids, the movement of Mexican bean beetles is affected by the

316 density of conspecifics. Experimental trials show that the probability of movement was

317 not affected by the number of conspecifics on the plant at densities lower than 20 beetles

318 per plant (Fig. 4b). However, dispersal rates went up rapidly when beetle density was

319 increased beyond 20 beetles per plant. Such behavior data suggest that at high population

320 densities, beetle movement becomes overdispersive. This example, together with the

321 others described earlier in this paper, highlights that density-dependent movement

322 behavior is a common, yet rarely unacknowledged, phenomenon in biology [90] and

323 ecology $[12,18,103,104]$, and its effects of spatial patterns formation and self-

324 organization needs further investigation.

\section{Indicators of phase separation dynamics in animals}

326 Although the model of Cahn and Hilliard can generate similar spatial patterns as those

327 predicted by models based on Turing's activator-inhibitor principle, there are several

328 crucial differences. First, phase separation is essentially mass conserving, as it only

329 involves movement or diffusive processes, at least when following the standard definition

330 and equations [35]. The activator-inhibitor principle is, to the contrary, not a mass

331 conserving process per se, as spatial variation in growth and mortality are the essential

332 processes. Secondly, Turing patterns exhibit a characteristic wavelength, or a number of

333 characteristic wavelengths, under constant environmental conditions. This is the

334 consequence of limitations in the scale of interaction between organisms [107], as the rate

335 of movement of water or nutrients is limited by physical restrictions. In contrast, patterns

336 arising from phase separation do not exhibit a characteristic wavelength, but pattern 
337 development is characterized by a continuously increasing wavelength, which follows a

338 power law relationship with time, referred to as the Lifshitz-Slyozov law [41, 108, 109].

339 This process is well known as coarsening. In other words, the phase separation process,

340 in mass-conserving systems, will not develop stationary patterns. However, this time-

341 dependent power law may collapse in the long term if other process come into play that

342 limit movement, such as in mussels where food competition limits the formation of very

343 large clusters [24].

344 Going beyond the movement-driven patterns elucidated here, the pattern coarsening that

345 is observed in the phase separation process can in principle also be found in activator-

346 inhibitor systems when the inhibitor process is global, e.g. when there are no physical

347 restrictions in the range of negative, inhibitory interactions between the organisms [107,

$348110,111]$. These conditions have been suggested for the FitzHugh-Nagumo (FHN) model

349 and for semiarid vegetation when competition for limiting water resources is global

350 [111]. However, for most ecosystems with regular patterns, such as those observed in arid

351 ecosystems, this is likely unrealistic, as there are physical limitations in the extent that

352 organisms can influence each other via the depletion of water. In systems with moving

353 animals, global limitation of plant growth via the interaction with animals is more easily

354 conceivable $[112,113]$. Whether mobile grazers can generate coarsening dynamics in

355 patchy plant-herbivore systems is an important topic for future study.

356 A negative effective diffusivity is a prerequisite for phase separation to occur in the

357 original Cahn-Hilliard model [24], as well as in later physical studies on abiotic particles

$358[32,35,48]$. This requirement, however, was broadened in recent physical studies

359 highlighting that a negatively density-dependent swimming speed is a sufficient condition 
360 for phase separation to occur in case of 'active Brownian particles', i.e. organisms [27, 28,

361 37]. In case of 'self-propelled active matter', physics theory highlights, a decreasing

362 motility with increasing local density will trigger a positive feedback, whereby an

363 increase of the local density of individual particles leads to local slowdown, causes

364 further accumulation of these particles. Hence, negative density dependence can cause

365 phase separation even without explicit taxis or other orientational processes [29, 49],

366 which is confirmed by both simulations [36, 50] and experiments [24, 51]. The

367 application of phase separation principles to the movement of larger organisms, as put

368 forward in this overview, will expand the scope of this novel theory beyond the field of

369 theoretical physics.

370 Classical, mass-conservative phase separation exhibits coarsening dynamics, but simple

371 modifications of the Cahn-Hilliard phase-separation model with a linear dissipative term

372 (i.e. mortality and birth processes in ecological terminology) may inhibit the coarsening

373 and lead to steady state patterns with a fix wavelength [46, 48]. Yet, a fundamental

374 difference exists between pattern formation as predicted by Turing's and Cahn-Hilliard's

375 principles [24]. The patterns arising from a Turing instability develop a homogeneous

376 background state, which becomes unstable with respect to small spatially perturbations,

377 leading the formation of regular patterns [114]. In phase separation, there is no such

378 unstable background state, but patterns arise from the spatial mixing of two stable states

379 (see Box 1 and Fig.5), creating patches of either high or low biomass. There are, in

380 general terms, only local attractive or repulsive forces among individuals that cause

381 biased movement in case of phase separation, and that drive the dynamic development of

382 the patterns. 
383 The difference between the two mechanisms becomes clearer when comparing the

384 feedback effects on net growth by the activator-inhibitor interaction with the effect of

385 local density on the effective diffusivity in the phase separation process. A switch

386 between activation and inhibition of either net local growth or movement characterize

387 both mechanisms (Fig. 5). However, in Turing's model this alternation occurs in response

388 to increased scale, while in the Cahn-Hilliard model this alternation occurs in response to

389 biomass or density (c.f Fig.5a and 5b). As a consequence, Turing-type systems adhere to

390 specific scales, while no characteristic scales are observed in phase separation.

391 Although many Turing models include explicit activator and inhibitor components [15,

392 42], an explicit configuration is not essential to generate a Turing instability. Spatial

393 patterns can also develop from perturbations of an unstable homogeneous state in single-

394 species systems, as is observed in the Swift-Hohenberg model, and in non-local

395 competition models [114-116]. In the past decades, non-local growth and interaction

396 effects have been proposed to produce observed regular spatial patterns in ecology and

397 biology [115-119], which consider non-local competition for resources in a variety of

398 biological fields $[120,121]$. In a population-dynamical context, these nonlocal growth

399 and interaction models have two features in common with Turing systems, i.e. growth-

400 and mortality- driven demographic processes and scale-depend feedback [2].

\section{Outlook}

402 Our review reveals that density-dependent effects on movement velocity and coordinated

403 turn frequency is a ubiquitous mechanism for the formation of self-organized spatial

404 patterns in ecosystems. Density-dependent movement, were speed declines over at least 
405 some range of local population density, can readily generate pattern formation in a wide

406 range of ecological settings. Moreover, density-dependent movement is broadly observed

407 in ecological systems, affecting the spatial dispersal of many organisms [103, 104, 122-

408 124]. Hence, the principle of density-dependent movement can be usefully extended to

409 other ecological systems that do not exhibit conspicuous regular patterns, but nonetheless

410 reveal more complex forms of aggregations, such as animal herds.

411 Many real ecosystems exhibit complex spatial organization, revealing patterns and

412 patches at multiple spatial scales. Such complexity does not typically stem from a single

413 underlying process. Multiple pattern-forming processes involving movement on short

414 time scales, and demographic and habitat-modifying processes at longer time scales are

415 superimposed to generate complexity. Little is known about how these processes interact

416 to shape ecosystems, and how this affects ecosystem functioning in terms of the

417 established population sizes, and their resilience against disturbances [46, 125]. Possibly,

418 the benefits of aggregation, in terms of for instance reduced predation risk, can affect

419 demographic process such as population losses. This has important implications, as by

420 influencing survival, movement-driven spatial patterns are likely to affect evolutionary

421 processes that in turn shape movement behavior [23]. Hence, to understand the

422 functioning of complex ecosystems, research should not focus on a single process driving

423 pattern formation, but should address how behavioral, demographic and evolutionary

424 processes interact.

\section{Acknowledgements}

426 We thank Dr. Heidi Fisher (Harvard University) for providing the photograph shown in 
427 Fig.3a and helpful comments on the ideas expressed of the sperm collective behavior.

428 This study was financially supported by the Netherlands Organization for Scientific

429 Research (NWO) through the project WaddenEngine of the National Programme Sea and

430 Coastal Research. Q.-X. L was partly supported by autonomous project of SKLEC 431 (2015RCDW01 and 2015KYYW03) and project of the thousand youth talents plan in 432 China. 


\section{Figure Captions:}

434 Fig 1: Observations of regular patterns from density dependent movement

435 processes. (a-b) Labyrinth patterns of small-scale mussel beds in intertidal flats (scale

436 about $50 \mathrm{~cm}$ ), and experimental data show that mussel-movement speed reveals a 437 quadratic function of mussel density. (c-d) Self-organized cluster formation in ants driven

438 by aggregation starting from a random initial condition. Their probabilities of dropping a 439 corpse remarkabley depend on the local density. The data come from experiments in Ref.

440 [18]. (e) Self-organized branching patterns in Dictyostelium discoideum. The cells 441 produce alternately lower and higher levels of cAMP concentrations as a two dimensional 442 spiral (scroll) waves from the mutant centre (later as aggregation center) to outwards, the

443 cells move inwards from flat territories. (f) Typical circular patterns obtained for the 444 engineered strain CL3, Escherichia coli [62] where the CL3 cells spotted at the center of 445 a semi-solid agar plate, scale bar about $1 \mathrm{~cm}$. Photo (a) by Johan van de Koppel; photos 446 (c) by Guy Theraulaz; photo (e) copyright Bioayuda in Microbiología, Noticias, 447 http://bioayuda.wordpress.com/tag/amebas-que-comen-bacterias; photo (f) copyright with 448 Science, 334, 2011, 238-241.

449 Fig 2: Density-dependent aggregation in mobile patterns. (a) An aggregation of elk 450 formed by density dependent movement. (b) Massive consumer front of wildebeest on 451 grasslands in Africa extending tens of kilometers in length and commonly more than 1

$452 \mathrm{~km}$ in width. (c-d) The daily probability of leaving a group versus group size on elk (error 453 bars shown 2 s.e. around each point); The probability of a solitary individual grouping up 454 with another elk versus the distance (error bars shown 2 s.e. around each point), where 
455 the fitted curve is a four-parameter exponential model, data from the Haydon et al. [75].

456 Photo (a) by John Fryxell; photo (b) copyright Serengeti Balloon Safaris, 457 http://www.balloonsafaris.com.

458 Fig 3: Denistiy-depedent collective behaviour of sperm cells of deer mice. (a)

459 Scanning electron micrographs of group formation of peromyscus sperm cells, startng

460 from initial release with highly motile (more than $90 \%$ progressively motile) single cells.

461 (b) The relaionship between aggregate size and average movement velocity of the sperm

462 cells of two species of deer mice. Figure and data courtesy of Heidi S. Fisher.

463 Fig 4: More evidence for density-dependent movement in ecology. (a) The probability

464 of stopping as a function of local aphid density, where the curve is a quadratic

465 polynomial fit, data come from Ref. [102]. (b) The density-dependent motility in the

466 Mexican bean beetle, data from Ref. [103].

467 Fig 5: Schematic representation of the feedback on the phase separation and the 468 activator-inhibitor principles. (a) The dispersion relation of the spatial pattern between 469 the effective diffusivity and species local density, where the two curves corresponding to 470 the lines in the inset panel. (b) The scale-dependent feedback in the activator-inhibitor 471 principles. 


\section{Box 1: The activator-inhibitor and phase separation principles}

The models of Turing (1953) and Cahn-Hilliard (1958) outline two fundamentally

474 different principles of spatial self-organization. Turing's model for pattern formation is based on the interaction between (minimally) two species, an activator and an

476 inhibitor (Box 1: Fig. A), which disperse at different rates in space. The interplay between the activator and the inhibitor results in spatial variation in net growth of or

478 rate of reaction between interacting species, which amplifies local variability, leading to the formation of spatial patterns. In contrast, the model Cahn and Hilliard for phase 480 separation and subsequent pattern formation only requires a single species, whose net movement switches between aggregation and dispersion as a function of its own local 482 density (Box 1: Fig. B).

\section{[Figure box at here]}

484 Fig I. (A) Alan Turing's (1912-1954) principle for spatial pattern formation, based on the interaction of an activator and an inhibitor. Here, the activator promotes it's own

486 growth, but also generates an inhibitor that limits activator growth. The two species diffuse through the system at different rates, with the inhibitor moving faster than the

488 activator. As a result, the activator has a net positive effect on itself at short distance, but inhibits itself at longer distance, via the inhibitor. (B) The Cahn-Hilliard principle

490 (John W Cahn, 1928-present; John E Hilliard, 1926-1987) for phase separation in pattern formation. This principle is based on density-dependent movement, where

492 species tend to disperse at low and very high density, but aggregate at intermediate density. The principle has been developed initially to explain self-organized patterns 494 in metal alloys, and formation of minerals. 


\begin{tabular}{llrlll}
\hline Ecosystem & $\begin{array}{l}\text { Spatial } \\
\text { mechanism }\end{array}$ & self-organization & Pattern formation & Refs \\
\hline Mussel & $\begin{array}{l}\text { Density-dependent } \\
\text { behavior }\end{array}$ & movement & Regular striped, labyrinth patterns & {$[7,12]$} \\
Ant & $\begin{array}{l}\text { Density-dependent } \\
\text { behavior }\end{array}$ & movement & Aggregation to clusters & {$[18]$} \\
Bacteria (E. coli) & $\begin{array}{l}\text { Density-dependent } \\
\text { behavior }\end{array}$ & chemo-taxis & Striped, spotted and circuits & {$[48,62,66]$} \\
Birds & $\begin{array}{l}\text { Resource-dependent } \\
\text { behavior }\end{array}$ & movement & Spatial regular patches & {$[68]$} \\
Elk (Cervus canadensis) & $\begin{array}{l}\text { Socially inform } \\
\text { Zebrafish }\end{array}$ & $\begin{array}{l}\text { Run-and-chase } \\
\text { behavior }\end{array}$ & movements & Striped or spotted patterns & {$[126]$} \\
\hline Sperm & $\begin{array}{l}\text { Integrated geometry } \\
\text { drag }\end{array}$ & with minima & Collective movement & {$[100]$} \\
\hline
\end{tabular}

Table 1: A summary of the movement-driven mechanisms on spatial self-organization in ecosystems. 
498

500

502

504

506

508

[1] Solé RV, Bascompte J. Self-organization in complex ecosystems. Princeton, NJ: Princeton University Press; 2006.

[2] Rietkerk M, Van de Koppel J. Regular pattern formation in real ecosystems. Trends Ecol Evol. 2008;23:169-75.

[3] Deblauwe V, Barbier N, Couteron P, Lejeune O, Bogaert J. The global biogeography of semi-arid periodic vegetation patterns. Global Ecology \& Biogeography. 2008;17:715-23.

[4] Nicolis G, Prigogine I. Self-organization in nonequilibrium systems: from dissipative structures to order through fluctuations. New York: Wiley; 1977.

[5] Klausmeier CA. Regular and irregular patterns in semiarid vegetation. Science. 1999;285:838-.

[6] Rietkerk M, Boerlijst MC, van Langevelde F, HilleRisLambers R, van de Koppel J, Kumar L, et al. Self-organization of vegetation in arid ecosystems. Am Nat. 2002;160:524-30.

[7] van de Koppel J, Rietkerk M, Dankers N, Herman PMJ. Scale-dependent feedback and regular spatial patterns in young mussel beds. Am Nat. 2005;165:E66-E77.

[8] van de Koppel J, Crain CM. Scale-dependent inhibition drives regular tussock spacing in a freshwater marsh. Am Nat. 2006;168:E136-E47.

[9] Liu Q-X, Weerman EJ, Herman PMJ, Olff H, van de Koppel J. Alternative mechanisms alter the emergent properties of self-organization in mussel beds. Proceedings of the Royal Society B-Biological Sciences. 2012;279:2744-53.

[10] Bonachela JA, Pringle RM, Sheffer E, Coverdale TC, Guyton JA, Caylor KK, et al. Termite mounds can increase the robustness of dryland ecosystems to climatic change. Science. 2015;347:651-5.

[11] Rietkerk M, Dekker SC, de Ruiter PC, van de Koppel J. Self-organized patchiness and catastrophic shifts in ecosystems. Science. 2004;305:1926-9.

[12] van de Koppel J, Gascoigne JC, Theraulaz G, Rietkerk M, Mooij WM, Herman PMJ. Experimental evidence for spatial self-organization and its emergent effects in mussel bed ecosystems. Science. 2008;322:739-42.

[13] Scheffer M, Bascompte J, Brock WA, Brovkin V, Carpenter SR, Dakos V, et al. Early-warning signals for critical transitions. Nature. 2009;461:53-9.

[14] Pringle RM, Doak DF, Brody AK, Jocque R, Palmer TM. Spatial Pattern Enhances Ecosystem Functioning in an African Savanna. Plos Biology. 2010;8.

[15] Turing AM. The Chemical Basis of Morphogenesis. Phil Trans R Soc Lond B. 1952;237:37-72. 
532 [16] Meinhardt H. Models of biological pattern formation. London ; New York: Academic Press; 1982.

534 [17] Meinhardt H, Fowler DR, Prusinkiewicz P. The algorithmic beauty of sea shells. 4th ed. Berlin; London: Springer,; 2009. p. xiv, 269p.

536 [18] Theraulaz G, Bonabeau E, Nicolis SC, Solé RV, Fourcassié V, Blanco S, et al. Spatial patterns in ant colonies. Proc Natl Acad Sci USA. 2002;99:9645-9.

538 [19] Quinn JL, Cresswell W. Testing domains of danger in the selfish herd: sparrowhawks target widely spaced redshanks in flocks. Proc R Soc Lond B.

$540 \quad$ 2006;273:2521-6.

[20] Ballerini M, Cabibbo N, Candelier R, Cavagna A, Cisbani E, Giardina I, et al.

542 Interaction ruling animal collective behavior depends on topological rather than metric distance: Evidence from a field study. Proceedings of the National Academy of

544 Sciences. 2008; 105:1232-7.

[21] Cavagna A, Cimarelli A, Giardina I, Parisi G, Santagati R, Stefanini F, et al.

546 Scale-free correlations in starling flocks. Proceedings of the National Academy of Sciences. 2010;107:11865-70.

548 [22] Folmer EO, Olff H, Piersma T. How well do food distributions predict spatial distributions of shorebirds with different degrees of self-organization? Journal of

550 Animal Ecology. 2010;79:747-56.

[23] de Jager M, Weissing FJ, Herman PMJ, Nolet BA, van de Koppel J. Levy Walks

552 Evolve Through Interaction Between Movement and Environmental Complexity.

Science. 2011;332:1551-3.

554 [24] Liu Q-X, Doelman A, Rottschafer V, de Jager M, Herman PMJ, Rietkerk M, et al. Phase separation explains a new class of self-organized spatial patterns in

556 ecological systems. Proceedings of the National Academy of Sciences of the United States of America. 2013;110:11905-10.

558 [25] Reynolds A. Liberating Lévy walk research from the shackles of optimal foraging. Physics of Life Reviews. 2015;14:59-83.

560 [26] de Jager M, Bartumeus F, Kölzsch A, Weissing FJ, Hengeveld GM, Nolet BA, et al. How superdiffusion gets arrested: ecological encounters explain shift from Lévy to

562 Brownian movement. Proceedings of the Royal Society B: Biological Sciences. $2014 ; 281$.

564 [27] Stenhammar J, Tiribocchi A, Allen RJ, Marenduzzo D, Cates ME. Continuum Theory of Phase Separation Kinetics for Active Brownian Particles. Physical Review

566 Letters. 2013;111:145702.

[28] Speck T, Bialké J, Menzel AM, Löwen H. Effective Cahn-Hilliard Equation for 568 the Phase Separation of Active Brownian Particles. Physical Review Letters. 2014;112:218304. 
570 [29] Cates ME, Tailleur J. Motility-Induced Phase Separation. Annual Review of Condensed Matter Physics. 2015;6:219-44.

572 [30] Fisher RA. The wave of advance of advntageous genes. Annals of Eugenics. 1937;7:355-69.

574 [31] Okubo A, Levin SA. Diffusion and ecological problems: mathematical models. Berlin; New York: Springer-Verlag; 1980.

576 [32] Cohen DS, Murray JD. A Generalized Diffusion-Model for Growth and Dispersal in a Population. J Math Biol. 1981;12:237-49.

578 [33] Levin S, Segel L. Pattern Generation in Space and Aspect. SIAM Review. $1985 ; 27: 45-67$.

580 [34] Grünbaum D. Translating stochastic density-dependent individual behavior with sensory constraints to an Eulerian model of animal swarming. J Math Biol.

$582 \quad 1994 ; 33: 139-61$.

[35] Cahn JW, Hilliard JE. Free Energy of a Nonuniform System 1 Interfacial Free

584 Energy. J Chem Phys. 1958;28:258-67.

[36] Redner GS, Hagan MF, Baskaran A. Structure and Dynamics of a Phase-

586 Separating Active Colloidal Fluid. Physical Review Letters. 2013;110:055701.

[37] Nguyen NHP, Daphne K, Michael E, Glotzer SC. Emergent collective

588 phenomena in a mixture of hard shapes through active rotation. Physical Review Letters. 2013;112:767-71.

590 [38] Sabrina SJM, Spellings M, Glotzer SC, Bishop KJM. Coarsening dynamics of binary liquids with active rotation. Soft Matter. 2015.

592 [39] Chevallard C, Clerc M, Coullet P, Gilli JM. Zig-zag instability of an Ising wall in liquid crystals. EPL (Europhysics Letters). 2002;58:686.

594 [40] Jasnow D, Viñals J. Coarse-grained description of thermo-capillary flow. Physics of Fluids. 1996;8:660-9.

596 [41] Bray AJ. Theory of phase-ordering kinetics. Advances in Physics. 2002;51:481587.

598 [42] Murray JD. Mathematical biology. 3rd ed. New York: Springer; 2002.

[43] Sumpter DJT. Collective Animal Behavior: Princeton University Press; 2010.

600 [44] Fick A. Ueber Diffusion. Ann der Physik. 1855;94:59.

[45] Einstein A. Investigations on the theory of Brownian Movement. Annalen der 602 Physik. 1905;17:549-60.

[46] Chevallard C, Clerc M, Coullet P, Gilli J-M. Interface dynamics in liquid

604 crystals. The European Physical Journal E. 2000;1:179-88. 
[47] Falk F. Cahn-Hilliard Theory and Irreversible Thermodynamics. J Non-Equil

606 Thermody. 1992;17:53-65.

[48] Cates ME, Marenduzzo D, Pagonabarraga I, Tailleur J. Arrested phase separation

608 in reproducing bacteria creates a generic route to pattern formation. Proc Natl Acad

Sci USA. 2010;107:11715-20.

610 [49] Tailleur J, Cates ME. Statistical Mechanics of Interacting Run-and-Tumble Bacteria. Physical Review Letters. 2008;100:218103.

612 [50] Fily Y, Marchetti MC. Athermal Phase Separation of Self-Propelled Particles with No Alignment. Physical Review Letters. 2012;108:235702.

614 [51] Buttinoni I, Bialké J, Kümmel F, Löwen H, Bechinger C, Speck T. Dynamical Clustering and Phase Separation in Suspensions of Self-Propelled Colloidal Particles.

616 Physical Review Letters. 2013;110:238301.

[52] Camazine S, Deneubourg J-L, Franks NR, Sneyd J, Theraulaz G, Bonabeau E.

618 Self-organization in biological systems. Princeton, N.J.; Oxford: Princeton University Press; 2001.

620 [53] Bonabeau E, Dorigo M, Theraulaz G. Swarm intelligence: from natural to artificial systems. New York: Oxford University Press; 1999.

622 [54] Tyler MS. Developmental biology: a guide for experimental study. Sunderland, Mass: Sinauer; 1994.

624 [55] Tyson JJ, Murray JD. Cyclic AMP waves during aggregation of Dictyostelium amoebae. Development. 1989;106:421-6.

626 [56] Pálsson E, Cox EC. Origin and evolution of circular waves and spirals in Dictyostelium discoideum territories. Proceedings of the National Academy of

628 Sciences. 1996;93:1151-5.

[57] Dormann D, Weijer CJ. Chemotactic cell movement during Dictyostelium

630 development and gastrulation. Current Opinion in Genetics \& Development. 2006;16:367-73.

632 [58] Dallon JC, Othmer HG. A discrete cell model with adaptive signalling for aggregation of Dictyostelium discoideum. Philos T R Soc B. 1997;352:391-417.

634 [59] van Oss C, Panfilov AV, Hogeweg P, Siegert F, Weijer CJ. Spatial pattern formation during aggregation of the slime mould Dictyostelium discoideum. Journal 636 of Theoretical Biology. 1996;181:203-13.

[60] Budrene EO, Berg HC. Dynamics of formation of symmetrical patterns by 638 chemotactic bacteria. Nature. 1995;376:49-53.

[61] Budrene EO, Berg HC. Complex patterns formed by motile cells of Escherichia 640 coli. Nature. 1991;349:630-3. 
[62] Liu C, Fu X, Liu L, Ren X, Chau CKL, Li S, et al. Sequential Establishment of

642 Stripe Patterns in an Expanding Cell Population. Science. 2011;334:238-41.

[63] Tsimring L, Levine H, Aranson I, Ben-Jacob E, Cohen I, Shochet O, et al.

644 Aggregation Patterns in Stressed Bacteria. Physical Review Letters. 1995;75:1859-62.

[64] Mittal N, Budrene EO, Brenner MP, van Oudenaarden A. Motility of Escherichia

646 coli cells in clusters formed by chemotactic aggregation. Proc Natl Acad Sci USA.

2003;100:13259-63.

648 [65] Brenner MP. Chemotactic patterns without chemotaxis. Proceedings of the National Academy of Sciences. 2010;107:11653-4.

650 [66] Fu X, Tang L-H, Liu C, Huang J-D, Hwa T, Lenz P. Stripe Formation in Bacterial Systems with Density-Suppressed Motility. Physical Review Letters.

$652 \quad 2012 ; 108: 198102$.

[67] Couzin ID, Krause J. Self-organization and collective behavior in vertebrates.

654 Adv Stud Behav. 2003;32:1-75.

[68] Folmer EO, Olff H, Piersma T. The spatial distribution of flocking foragers:

656 disentangling the effects of food availability, interference and conspecific attraction by means of spatial autoregressive modeling. Oikos. 2012;121:551-61.

658 [69] Handegard Nils O, Boswell Kevin M, Ioannou Christos C, Leblanc Simon P, Tjøstheim Dag B, Couzin Iain D. The Dynamics of Coordinated Group Hunting and

660 Collective Information Transfer among Schooling Prey. Current biology. 2012;22:1213-7.

662 [70] Ioannou CC, Guttal V, Couzin ID. Predatory fish select for coordinated collective motion in virtual prey. Science. 2012;337:1212-5.

664 [71] Bender LC, Haufler JB. Social Group Patterns and Associations of Nonmigratory Elk (Cervus elaphus) in Michigan. The American Midland Naturalist. 1999;142:8766695.

[72] Jenkins KJ, Starkey EE. Social Organization of Roosevelt Elk in an Old-Growth 668 Forest. Journal of Mammalogy. 1982;63:331-4.

[73] Fryxell JM, Hazell M, Börger L, Dalziel BD, Haydon DT, Morales JM, et al.

670 Multiple movement modes by large herbivores at multiple spatiotemporal scales. Proceedings of the National Academy of Sciences. 2008;105:19114-9.

672 [74] Yott A, Rosatte R, Schaefer JA, Hamr J, Fryxell J. Movement and Spread of a Founding Population of Reintroduced Elk (Cervus elaphus) in Ontario, Canada.

674 Restoration Ecology. 2011;19:70-7.

[75] Haydon DT, Morales JM, Yott A, Jenkins DA, Rosatte R, Fryxell JM. Socially

676 informed random walks: incorporating group dynamics into models of population spread and growth. Proceedings of the Royal Society B: Biological Sciences.

$678 \quad 2008 ; 275: 1101-9$. 
[76] Anderson TM, Hopcraft JGC, Eby S, Ritchie M, Grace JB, Olff H. Landscapescale analyses suggest both nutrient and antipredator advantages to Serengeti herbivore hotspots. Ecology. 2010;91:1519-29.

682 [77] Gueron S, Levin SA. Self-organization of front patterns in large wildebeest herds. Journal of Theoretical Biology. 1993;165:541-52.

684 [78] Holdo RM, Holt RD, Fryxell JM. Opposing Rainfall and Plant Nutritional Gradients Best Explain the Wildebeest Migration in the Serengeti. Am Nat.

686 2009;173:431-45.

[79] Mloszewski MJ. The behavior and ecology of the African buffalo. Cambridge

688 Cambridgeshire; New York: Cambridge University Press; 1983.

[80] Hopcraft JGC, Morales JM, Beyer HL, Borner M, Mwangomo E, Sinclair ARE, 690 et al. Competition, predation, and migration: individual choice patterns of Serengeti migrants captured by hierarchical models. Ecological monographs. 2014;84:355-72.

692 [81] Gueron S, Levin SA, Rubenstein DI. The dynamics of herds: from individuals to aggregations. Journal of Theoretical Biology. 1996;182:85-98.

694 [82] Hopcraft JGC, Anderson TM, Perez-Vila S, Mayemba E, Olff H. Body size and the division of niche space: food and predation differentially shape the distribution of

696 Serengeti grazers. Journal of Animal Ecology. 2012;81:201-13.

[83] Hopcraft JGC, Olff H, Sinclair ARE. Herbivores, resources and risks: alternating 698 regulation along primary environmental gradients in savannas. Trends Ecol Evol. 2010;25:119-28.

700 [84] Sinclair ARE. The African buffalo: a study of resource limitation of populations: University of Chicago Press Chicago; 1977.

702 [85] Fryxell JM, Mosser A, Sinclair ARE, Packer C. Group formation stabilizes predator-prey dynamics. Nature. 2007;449:1041-3.

704 [86] Fryxell JM, Greever J, Sinclair ARE. Why are migratory ungulates so abundant? Am Nat. 1988;131:781-98.

706 [87] Brian RS, McCoy MW, Angelini C, Holt RD, Griffin JN, van de Koppel J. Consumer Fronts, Spatial Processes, and Ecosystem Structure and Stability. Annual

708 Review of Ecology, Evolution, and Systematics. 2013;44:503-38.

[88] Silliman BR, van de Koppel J, Bertness MD, Stanton LE, Mendelssohn IA.

710 Drought, Snails, and Large-Scale Die-Off of Southern U.S. Salt Marshes. Science. 2005;310:1803-6.

712 [89] Lauzon-Guay J-S, Scheibling RE. Spatial dynamics, ecological thresholds and phase shifts: modelling grazer aggregation and gap formation in kelp beds. Mar Ecol

714 Prog Ser. 2010;403:29-41. 
[90] Farrell FDC, Marchetti MC, Marenduzzo D, Tailleur J. Pattern Formation in

716 Self-Propelled Particles with Density-Dependent Motility. Physical Review Letters. 2012;108:248101.

718 [91] Buhl J. From disorder to order in marching locusts. Science. 2006;312:1402-6.

[92] Szabó B, Szöllösi GJ, Gönci B, Jurányi Z, Selmeczi D, Vicsek T. Phase

720 transition in the collective migration of tissue cells: Experiment and model. Physical Review E. 2006;74:061908.

722 [93] Trepat X, Wasserman MR, Angelini TE, Millet E, Weitz DA, Butler JP, et al. Physical forces during collective cell migration. Nature Physics. 2009;5:426-30.

724 [94] Méhes E, Mones E, Németh V, Vicsek T. Collective Motion of Cells Mediates Segregation and Pattern Formation in Co-Cultures. PLoS ONE. 2012;7:e31711.

726 [95] Basan M, Elgeti J, Hannezo E, Rappel WJ, Levine H. Alignment of cellular motility forces with tissue flow as a mechanism for efficient wound healing.

728 Proceedings of the National Academy of Sciences of the United States of America. 2013;110:2452-9.

730 [96] Buhl J, Sumpter DJT, Couzin ID, Hale JJ, Despland E, Miller ER, et al. From Disorder to Order in Marching Locusts. Science. 2006;312:1402-6.

732 [97] Gaffney E, Gadêlha H, Smith DJ, Blake JR, Kirkman-Brown J. Mammalian sperm motility: observation and theory. Annual Review of Fluid Mechanics.

$734 \quad 2011 ; 43: 501-28$.

[98] Immler S. Sperm competition and sperm cooperation: the potential role of

736 diploid and haploid expression. Reproduction. 2008;135:275-83.

[99] Pizzari T, Foster KR. Sperm sociality: cooperation, altruism, and spite. PLoS 738 biology. 2008;6:e130.

[100] Fisher HS, Giomi L, Hoekstra HE, Mahadevan L. The dynamics of sperm

740 cooperation in a competitive environment. Proceedings of the Royal Society B: Biological Sciences. 2014;281:20140296.

742 [101] Fisher HS, Hoekstra HE. Competition drives cooperation among closely related sperm of deer mice. Nature. 2010;463:801-3.

744 [102] Aronson DG. Density-Dependent Interaction-Diffusion Systems. Dynamics \& Modelling of Reactive Systems. 1980:161-76.

746 [103] Turchin P, Kareiva P. Aggregation in Aphis Varians: An Effective Strategy for Reducing Predation Risk. Ecology. 1989;70:1008-16.

748 [104] Turchin P. Population Consequences of Aggregative Movement. Journal of Animal Ecology. 1989;58:75-100.

750 [105] Kuefler D, Avgar T, Fryxell JM. Density- and resource-dependent movement characteristics in a rotifer. Funct Ecol. 2013;27:323-8. 
752 [106] Kuefler D, Avgar T, Fryxell JM. Rotifer population spread in relation to food, density and predation risk in an experimental system. Journal of Animal Ecology.

$754 \quad 2012 ; 81: 323-9$.

[107] Kletter AY, Von Hardenberg J, Meron E. Ostwald ripening in dryland vegetation. Communications on Pure \& Applied Analysis. 2012;11:261-73.

[108] Lifshitz IM, Slyozov VV. The kinetics of precipitation from supersaturated solid solutions. Journal of Physics and Chemistry of Solids. 1961;19:35-50.

[109] Argentina M, Clerc MG, Rojas R, Tirapegui E. Coarsening dynamics of the

760 one-dimensional Cahn-Hilliard model. Physical Review E. 2005;71:046210.

[110] von Hardenberg J, Kletter AY, Yizhaq H, Nathan J, Meron E. Periodic versus

762 scale-free patterns in dryland vegetation. Proceedings of the Royal Society BBiological Sciences. 2010;277:1771-6.

764 [111] Meron E. Nonlinear physics of ecosystems. London, New York: CRC, Taylor \& Fancis Goup; 2015.

766 [112] Kefi S, Rietkerk M, Alados CL, Pueyo Y, Papanastasis VP, ElAich A, et al. Spatial vegetation patterns and imminent desertification in Mediterranean arid

768 ecosystems. Nature. 2007;449:213-U5.

[113] Berg G, Esselink P, Groeneweg M, Kiehl K. Micropatterns in Festuca rubra-

770 dominated salt-marsh vegetation induced by sheep grazing. Plant Ecology. 1997;132:1-14.

772 [114] Cross M, Greenside H, ebrary Inc. Pattern formation and dynamics in nonequilibrium systems. Cambridge, UK ; New York: Cambridge University Press; 7742009.

[115] Fuentes MA, Kuperman MN, Kenkre VM. Nonlocal Interaction Effects on

776 Pattern Formation in Population Dynamics. Physical Review Letters. 2003;91:158104.

778 [116] Fuentes MA, Kuperman MN, Kenkre VM. Analytical Considerations in the Study of Spatial Patterns Arising from Nonlocal Interaction Effects. The Journal of

780 Physical Chemistry B. 2004;108:10505-8.

[117] da Cunha JAR, Penna ALA, Oliveira FA. Pattern formation and coexistence 782 domains for a nonlocal population dynamics. Physical Review E. 2011;83:015201.

[118] Clerc MG, Escaff D, Kenkre VM. Patterns and localized structures in 784 population dynamics. Phys Rev E. 2005;72:056217.

[119] Kenkre VM. Patterns, Noise, and the Interplay of Nonlinearity and Complexity.

786 In: Kenkre VM, Lindenberg K, editors. Modern Challenges in Statistical Mechanics. Bariloche (Argentina): AIP; 2003.

788 [120] Kenkre VM. Results from variants of the Fisher equation in the study of epidemics and bacteria. Physica A. 2004;342:242-8. 
790 [121] da Cunha JAR, Penna ALA, Vainstein MH, Morgado R, Oliveira FA. Selforganization analysis for a nonlocal convective Fisher equation. Physics Letters A.

792 2009;373:661-7.

[122] Bitume EV, Bonte D, Ronce O, Bach F, Flaven E, Olivieri I, et al. Density and 794 genetic relatedness increase dispersal distance in a subsocial organism. Ecol Lett. 2013;16:430-7.

796 [123] Cote J, Clobert J. Social personalities influence natal dispersal in a lizard. Proceedings of the Royal Society B: Biological Sciences. 2007;274:383-90.

798 [124] Ims RA, Andreassen HP. Density-dependent dispersal and spatial population dynamics. Proc R Soc B. 2005;272:913-8.

800 [125] Liu QX, Herman PMJ, Mooij WM, Huisman J, Scheffer M, Olff H, et al. Pattern formation at multiple spatial scales drives the resilience of mussel bed

802 ecosystems. Nature Communications. 2014;5:7.

[126] Yamanaka H, Kondo S. In vitro analysis suggests that difference in cell

804 movement during direct interaction can generate various pigment patterns in vivo. Proceedings of the National Academy of Sciences. 2014;111:1867-72. 